\title{
Cuerpo de una violencia infame. Algunas apreciaciones para una hermenéutica de la violencia
}

\author{
Body of an infamous violence. \\ Some insights for a hermeneutics of violence
}

YUBER HERNANDO ROJAS ARIZA*

\begin{abstract}
Resumen: La mirada sobre el cuerpo ha cambiado en el tiempo y ha sido múltiple como la violencia. El presente texto tiene como objetivo comprender el paso de la violencia sobre el cuerpo al cuerpo de la violencia. Para hacer efectivo el análisis, el texto se divide de la siguiente manera: 1) Breve compilación de la mirada de la Grecia antigua y medieval; 2) El cuerpo como objeto-técnico-científico en la época moderna; y 3) el cuerpo de la violencia en relación a la técnica. Con todo esto, finalmente se busca vislumbrar las posibilidades de una hermenéutica de la violencia para la comprensión de nuestros tiempos.
\end{abstract}

Palabras claves: Cuerpo, objeto-técnico-científico, violencia, Hermenéutica.

\begin{abstract}
Summary: The regard on the body has changed over time and has been multiple like the violence. This paper aims to interpret the transition from violence on the body to the body of violence. To give effect to the analysis, the text is divided as follows: 1) Short compilation of the ancient Greece and medieval interpretation; 2) The body as technical-scientific-object in modern times; and 3) the body of violence in relation to the technique. With all this, finally you can preview the possibilities of a hermeneutics of violence to understand our times.
\end{abstract}

Keywords: Body, Technical-scientific-object, Violence, Hermeneutics.

\section{Introducción}

La vieja potencia de la muerte, en la cual se simbolizaba el poder soberano, se halla ahora cuidadosamente recubierto por la administración de los cuerpos y la gestión calculadora de la vida.

(Michel Foucault).

De especial interés filosófico las múltiples violencias con las cuales se inaugura el siglo XXI. Se requiere una re-interpretación que permita comprender su relación con la vida moderna. La relación estrechamente ligada entre la Razón humana y la violencia sofisticada

Fecha de recepción: 01/07/2016 Fecha de aceptación: 06/09/2016.

* Economista, Filósofo, Mg. Filosofía, Universidad Industrial de Santander UIS. Profesor Universidad Pontificia Bolivariana, UPB (Bucaramanga-Colombia). 
de nuestros tiempos se patenta en la vida cotidiana. Tan habituado está el hombre moderno a la violencia que emerge de su propia razón que no se detiene por un instante a reflexionar sobre sus profundas implicaciones, no se detiene, para el caso, a re-interpretar la noción de Cuerpo y su íntima relación con lo político.

La siguiente propuesta de estudio se centrará en el cuerpo de la violencia infame. O para ser más explícito vale resaltar la pregunta: ¿Cómo se ha pasado de la violencia del cuerpo a una especie del cuerpo de la violencia? Aquí Cuerpo-Violencia se convierte en el epicentro o si quiere, en el vehículo del texto. Una hermenéutica de la violencia implica una comprensión del cuerpo de la misma: ¿Cuál es entonces el cuerpo de la violencia? Para poder atender a dicha pregunta es importante entender que tal estado ha tenido una transformación en el tiempo y que se requiere ver con especial cuidado.

En efecto se plantea lo siguiente: en primer lugar, entender el cuerpo en su relación originaria, a saber, el cuerpo como un Sujeto-todo-vivo. En segundo lugar, me centraré en la visión moderna: el cuerpo como Objeto-técnico-científico. El cuerpo, entendido de esa forma, se ha convertido en un territorio del conocimiento técnico-científico. Un tercer y último momento del texto plantea la posibilidad del cuerpo humano en su expresión necrológica: la comprensión de la Techné moderna sobre el cuerpo -o sobre el cuerpo de la violencia-, cuyo estado se encuentra atado al Régimen disciplinario de la vida moderna; una lógica al servicio de la muerte donde el conocimiento humano resulta determinante.

Las distintas guerras constatan aquello. Aquí entra en consideración la necesaria indagación sobre una especie de Hermenéutica de la violencia fundamentada en la condición necrológica del cuerpo humano. Luego, frente a la relación Cuerpo-política, el presente texto propone pensar en la transformación de la violencia del cuerpo al cuerpo de la violencia. De allí que en la última parte de la propuesta permita pensar la violencia (humana) a partir de la Techné "moderna": el cuerpo en medio de la violencia infame que inaugura el siglo XXI.

\section{Precedentes sobre el cuerpo humano: del Sujeto-todo-vivo al Objeto-del-pecado}

La interpretación del cuerpo ha mutado en el tiempo. Me propongo que nos aproximemos a la comprensión de ese devenir sobre el cuerpo. Quiero que nos centremos en las dos visiones más importantes que obedecen, claro está, a dos posturas muy distintas del mundo. La primera tendrá como referencia la visión griega antigua; y la segunda la visión medieval. Para ello sugiero que nos des-hagamos de nuestros prejuicios, que los tengamos suspendidos por un momento, para así lograr indagar sobre las características de dichas interpretaciones sobre el cuerpo.

\subsection{La visión antigua: el cuerpo como un Sujeto-todo-vivo}

Aunque la palabra "Sujeto" tiene una carga moderna muy alta, considero que puede ser útil para tratar de entender esa idea sobre todo-vivo. Cuando hablamos de cuerpo en la Grecia antigua lo primero que se debe tener en cuenta es su condición estética: el canon de belleza; y lo segundo, no menos importante, tiene que ver con su condición física; el canon de naturaleza. Tanto uno y otro canon establecen dos conexiones del cuerpo: belleza- 
naturaleza. El cuerpo humano, en ese sentido, es sumamente importante para los griegos en la comprensión del mundo, sobre todo porque de allí emerge un culto a la figura humana y también a la figura divina. Una y otra figura, a veces amorfas, otras veces con un relieve humanizado, representa, sin embargo, en ese reino mitológico o divino, la forma como veían el mundo, el universo. Digamos que eso contribuye notoriamente a la Poiesis, a la creación artística. Referente de la literatura, la poesía, la escultura, la arquitectura e inclusive de la filosofía. Todas estas expresiones del conocimiento humano con un solo referente: la forma del cuerpo humano.

Pero quiero que nos detengamos por un momento en eso que he llamado todo-vivo: el cuerpo como sujeto. Si se me permite la aseveración: el cuerpo humano en la época antigua tiene esa característica. Bien sabido cómo el cuerpo resultaba importante para la guerra o inclusive para la fertilidad; o bien sabido, igualmente, el legado de los egipcios en la cultura griega sobre la el mundo del "más allá"; o bien sabido, también, el cuerpo dentro de las constelaciones mito-lógicas. Quiero entonces establecer una atrevida pero quizás contundente manera de entender un poco más todo esto. En ese orden de exposición, significa la comprensión del cuerpo como un Sujeto- todo-vivo que se expresa al menos de tres formas: i) Orgánico-funcional (Reproducción-Guerra-Trabajo); ii) Estético-mágico (Trascendencia del Alma); y iii) todo esto apuntado, en síntesis a entender el Cuerpo como Medium del Espíritu.

Veamos con atención la primera forma: Orgánico-funcional. Esa primera forma de concebir el cuerpo busca asegurar la reproducción, ganar la guerra y servir para el trabajo. Luego Reproducción-guerra-trabajo comparten el común denominador en la Polis porque se refieren a tres ámbitos de la vida cotidiana de la Grecia antigua: biológico-político-económico. El común denominador de éste ámbito es el ciudadano de la Polis, aquel que pertenece a la Comunidad: el ser griego. En ese sentido, la primera forma orgánico-funcional busca conservar el ser griego. El cuerpo bajo ésta forma entonces identifica al ser griego: por su "raza" pero sobre todo para la Polis.

Si la primera forma tiene ese carácter funcional, utilitario, la segunda forma se centra en algo que lo complementa: el carácter Estético-mágico. Indica entonces la trascendencia del Alma. La época antigua (griega) tiene ese rasgo que permite afirmarlo. Platón cree, por ejemplo en la re-encarnación. La fuerte influencia del antiguo Egipto es notoria. La pregunta por el más allá, se traduce en Platón como la búsqueda de la Verdad. El encuentro con la luz, salir de la Caverna (Platón, 1992: 514a) Y más diciente en el Fedon cuando argumenta a favor de la inmortalidad del alma: por su carácter cíclico del cambio (69e6-72d10), por la afinidad del alma con las Ideas (78b4-84b7) y termina entonces con el argumento central sobre la sabiduría y la muerte de Sócrates (Platón: 102a10-107b10). Desde luego, más allá de esto, encontramos eso mágico del cuerpo: el alma inmortal. En resumidas cuentas: el cuerpo se prepara para la muerte, se cultiva con la sabiduría.

Muy cercana a la segunda forma, considero que la última es reveladora bajo la pregunta por el sentido del cuerpo: el cuerpo como Medium del "espíritu" de la Polis. El sentido del cuerpo se logra develar como parte viva de la Polis. O en otras palabras: la Polis como un sujeto-todo-vivo. Si lográramos comprender la dimensión de esto, entonces nos podríamos pre-ocupar un poco más por el cuerpo humano como un todo-vivo que también es político en nuestro tiempo. En síntesis, lo que quiero dejar en discusión es precisamente la visión 
reveladora de la Grecia antigua sobre el cuerpo a partir de las tres formas que he mencionado. Quizás haciendo especial énfasis en la última como un Sujeto-todo-vivo.

\subsection{La "transitoria" visión medieval: el cuerpo como objeto-del-pecado}

Pero el asunto se ha transformado en nuestro tiempo. Después de los griegos lo que viene es cómo se doblega el cuerpo, se busca el control sobre el mismo con fines de explotación en nombre de un ente supremo: dios. La época medieval es quizás la época más represiva sobre el cuerpo humano. En nombre de dios el cuerpo es doblegado, considerado un ente móvil de un ente supremo. La iglesia lo considera corruptible en tanto que el Mal puede apoderarse y subyugarlo. A esto se le llamó Pecado. Cuerpo-Pecado, la carne corruptible. El cuerpo pierde su valor de Sujeto-todo-vivo y comienza a transformarse en una especie de ente-corruptible-pecado. En efecto, la lectura que hace el filósofo Michel Foucault es acertada en esto. Por ejemplo, se castiga el placer porque es la manifestación del Mal; la sexualidad queda en manos de la Iglesia y su método confesionario; inquietante manifestación estudiada, por ejemplo, por Michel Foucault en Historia de la Sexualidad. Quien cometía "pecado" debía confesarse con el sacerdote. Un control del cuerpo muy agudo: la auto-prohibición del placer. También se constata esto en Las Confesiones de San Agustín sobre las pasiones (San Agustin: Libro VI).

Aunque hubo más represión que incentivo como mecanismos de control, no se puede dejar de lado que el cuerpo, su energía vital, quedaba entonces al servicio del trabajo. Tal vez sea el incentivo más predominante, la razón por la cual se permitía el sometimiento del cuerpo a los dictámenes de las leyes judeocristianas. Me refiero a dejar el cuerpo, su energía vital al servicio de dios: de la bondad que se creía emanaba del gran creador a través de la Iglesia. Todo esto se materializó en el trabajo. El cuerpo como ente productivo al servicio de la Ciudad de Dios. De allí que el cuerpo doblegado por las leyes judeocristianas quede al servicio de dos dictámenes: el castigo al pecado y el premio o incentivo por su trabajo al servicio de Dios. El gran referente fundacional: el pecado original (San Agustín, 1985: libro XIII y XIV). En ese sentido se puede re-interpretar la obediencia cristiana: la búsqueda de la redención. El goce en el más allá con la obediencia en el más acá: "por el árbol de la vida, en santo de los santos, que es Jesucristo, y por el árbol de la ciencia del bien y el mal, el propio albedrío de la voluntad, pues ni aun de sí mismo puede el hombre usar sino muy mal si desprecia la voluntad divina." (San Agustín: p. 86).

Sin embargo, esa voluntad divina se pone en cuestión. No en sí misma, sino más bien a la Iglesia como garante de tal voluntad. La visión que consideró el cuerpo como objeto del pecado se vio transformada por una visión que re-orientó el valor del cuerpo humano. La explosión del renacimiento, en la Italia de los grandes pintores y escultores y de la literatura de Dante, entre otras manifestaciones donde se constata el punto de quiebre, evidencia la ruptura con la visión medieval. La visión antropocéntrica tiene ese rasgo particular: el cuerpo humano como centro del Universo. Comienza a delinearse sus curvas y relieves, su color, sus músculos, huesos y carnes. El movimiento del cuerpo empieza a tener protagonismo. La estética aparece de nuevo para sobre-ponerse a lo religioso: el arte religioso hace explosión, salvo que esta vez es el hombre el protagonista y no dios. No es su negación, es más bien su re-afirmación. El cuerpo, en efecto, adquiere esa tonalidad positiva que le había sido 
arrebatada por la visión dogmática medieval. El arte revela la belleza divina a través de la grandeza del cuerpo humano.

\section{La visión moderna: el cuerpo como Objeto-técnico-científico}

Toda la propuesta del renacimiento también cambió para darle paso al cuerpo humano como objeto-técnico-científico. Aquello que los Antiguos consideraron un sujeto-todo-vivo proyectado en la Polis y que posteriormente pasó a convertirse en el medioevo en el cuerpo como objeto-del-pecado y proyecto en ciudad de Dios, ahora, en la época moderna, se transforma en un Objeto a merced del proceso técnico-científico. Significa que el cuerpo se ha transformado en un Objeto que actúa como un todo-mecánico. Desde luego, esa perspectiva de mecanización es expresión de nuestra tradición que lo considera objeto de explotación. Por consiguiente, significa que el Cuerpo como Objeto se expresa de varias formas: Primero como materia inerte (o ente-instrumental); segundo como Rendimiento y Productividad; y tercero, en su forma de Exhibición (consumo). Tres rasgos que explican una estética del Espectáculo y la lógica económica sobre el cuerpo en nuestros días.

\subsection{El cuerpo como Objeto: ente-instrumental}

El cambio más importante tiene que ver precisamente con esto. El cuerpo no solamente se transformó en un "Objeto de estudio" sino que también se transformó en un "Objeto-sinvida": materia inerte. Esa característica del cuerpo en la época moderna emana justamente del conocimiento técnico-científico. Así como se hizo patente en la biología y principalmente en la medicina, el cuerpo perdió en las otras "áreas del conocimiento" ese estatus vital. Dejó de ser vida para transformarse en un objeto de muerte. E inclusive los avances en la medicina solamente fueron posibles gracias al conocimiento que se obtenía de cuerpos sin vida. La morgue, por ejemplo, se convirtió en el gran laboratorio del conocimiento durante varios siglos, en particular desde el siglo XV hasta hoy en día en las universidades y facultades de medicina del siglo XX y XXI.

Lo sorpresivo y el cambio drástico que nos debe quedar claro justamente, tiene que ver con la pre-suposición del cuerpo como ente-instrumental, esto es, al servicio del avance técnico-científico moderno. Podrá decirse que sin esto no era posible el avance de la medicina o de la biología, o más recientemente de la neurología, pero lo que quiero señalar no es desconocer aquello sino más bien reconocer cómo se fue configurando una suposición sobre el cuerpo basado en un ente-instrumental. Ese carácter propio del conocimiento técnicocientífico ha irrumpido en otras "áreas del conocimiento" bajo otros nombres. Por ejemplo: en la economía se traduce en "cifras" gracias a la estadística, en la política se expresa en "votos" verbi gracia al sistema democrático. En general, trato de decir que el Quatum, el hecho contable de los cuerpos, convirtió el cuerpo humano en un simple número, en una abstracción, en algo carente de vida, frío, etéreo, completamente desprendido de su propia conexión vital.

Se desprende de todo esto algo que no puedo dejar de señalar en aras de comprender la tipología propuesta. Considerar el cuerpo como ente-instrumental a servicio del conocimiento técnico-científico cuya máximo sostén se basa en el Quatum es precisamente el 
cambio más importante en la época moderna. No se trata de una mera observación carente de cualquier sentido. Es quizás la más devastadora visión y la imposibilidad del reconocimiento entre los mismos humanos. Si antes podía entenderse el papel del cuerpo en la vida política de la Polis o si antes también se podía entender la obediencia con el sometimiento del cuerpo y sus respectivas prohibiciones, en la época moderna, en cambio, el problema de pre-suponer el cuerpo como materia inerte, indica que el sentido por la Humanidad se ve seriamente amenazado por el mismo hombre, a saber, por eso que denominamos con tanta ilusión avance técnico-científico.

\subsection{El cuerpo como Objeto: Rendimiento-productivo}

No obstante, lo anterior no tendría sentido sin considerar también el cuerpo como un ente de Rendimiento-productivo. Otra característica fundamental -desde luego porque tiene que ver con la vida- es el trabajo humano. No solamente nacemos, crecemos, nos reproducimos y morimos. También trabajamos. El trabajo humano es otra actividad del Cuerpo que en la época moderna adquiere una dimensión instrumental y absorbe gran parte de la energía vital. Significa que el cuerpo se transforma en un ente de explotación. Estamos sanos para trabajar, estamos listos para el trabajo. En la vida moderna, en especial bajo el canon del sistema productivo, hacer parte de la Población económica activa (PEA) se puede traducir en lo que se considera la "vida útil" del cuerpo. Por ejemplo, si pasas los 60 años, significa que ya has pasado el límite, es decir, ya no eres útil. Llega la pensión -en el mejor de los casos- como recompensa por los años de trabajo lo cual -permítaseme la expresión- quiere decir que tu cuerpo ya no sirve al sistema; y al final, lo que sigue, es pagar un dinero para cuidar la salud del cuerpo, lo poco que queda.

Lo anterior, evidentemente, deja al descubierto la visión del cuerpo como objeto en su versión de rendimiento-productivo. Veamos con más detalle la salud del cuerpo que es considerado inútil al sistema productivo. La salud, el cuidado del cuerpo explotado, es simplemente lo que se ha perdido: lo irrecuperable para el cuerpo y el dinero recuperable por el sistema productivo. Trabajar para enfermar en la vejez, y en la vejez pagar para obtener la salud que fue expropiada del cuerpo. Es una explotación del cuerpo: ¡el sistema productivo exprime hasta los cuerpos que considera inútiles! En efecto, nos permite el ejemplo real y concreto del mundo moderno, entender que la consideración del cuerpo como objetorendimiento-productivo se materializa en el trabajo; en lo cotidiano, en la contabilidad de 8 horas diarias consagradas al aumento de la productividad pero, de manera inversamente proporcional, equivalente a la disminución de la vitalidad. Es una aceleración de vida, un paso cada vez más rápido a la muerte.

\subsection{El cuerpo como Objeto de Exhibición-consumo}

No obstante, si lo anterior puede parecer revelador, no menos nos puede parecer éste. El cuerpo también se convirtió en un objeto de consumo. No solamente en la perspectiva económica donde el tiempo pareciera ser su principal corrosivo, el que "consume" la energía. Más bien quiero resaltar que el cuerpo ha adquirido una medida estándar, una producción serial de cómo-verse-en-el-mundo. Es una especie de estética para el consumo. Por supuesto, 
un canon impuesto muy ligado a las dos formas de concebir el cuerpo ya mencionadas. El cuerpo de la forma aquí expuesta, como exhibición-consumo, es un objeto que queda dispuesto al canon serial, estándar, empaquetado, envuelto en valores simbólicos de belleza. La gran novedad de la época moderna es que es un objeto no un sujeto el que posa, el que se exhibe. Lo novedoso es justamente la expresión más acabada de la apariencia sin el más sentido -si siguiera fielmente a Platón- de la esencia.

El cuerpo planteado de esa forma, precisamente se preocupa por su forma. Pero no es algo que sea causado por una visión fundamentada en el conocimiento de sí mismo. No. Obedece a la apariencia y, sobre todo, al estándar que se considera bello dentro del mundo moderno. El cuerpo volcado a la exhibición-consumo se convierte en la ilusión, en un estilo de vida, en una cotidianidad volcada a la apariencia, al sometimiento de los cada vez más sofisticados y exigentes cánones de belleza. Es una especie de rendimiento sobre la belleza frágil y fugaz: la apariencia de joven, cada vez más joven. Una contradicción que se busca -o anhela- resolver: una especie de la búsqueda de la "eterna juventud" del cuerpo.

Pero no hay cuerpo que lo soporte. La búsqueda de la "eterna juventud" y su contradicción con el deterioro inevitable de la condición biológica se convierte en un problema fundamental. El espectáculo del cuerpo se encuentra allí, en esa contradicción. Se vende, se consume, se exhibe y muere. Luego, el cuerpo en su versión exhibición-consumo llega a su máxima expresión en el mundo del espectáculo de la cotidianidad: el cuerpo como atuendo, como objeto para exhibir cosas, novedades, simplemente moda. En consecuencia, su máxima expresión pero a la vez su profunda crisis aflora en el espectáculo cuando el cuerpo se transforma en una mera vitrina andante, en un objeto más dentro de la sociedad de consumo. En esa perspectiva, la crisis del cuerpo salta a la vista y la carencia que padece ese espectáculo se agudiza cuando se demanda más contenido que forma.

\section{Techné moderna sobre el cuerpo: algunas apreciaciones sobre el Cuerpo de la violencia}

Las tres formas del cuerpo como objeto en la época moderna tienen su gran síntesis en la violencia. Es realmente revelador cuando cuerpo y violencia se fusionan. Significa que la interpretación de la relación cuerpo-objeto que caracteriza la época moderna adquiere su máxima expresión cuando se logra ver quizás su más bella y a la vez fría fundamentación: la relación cuerpo-violencia. El breve recorrido por la transformación del cuerpo adquiere sentido hoy en día cuando comprendemos el paso de la violencia del cuerpo materializada por la relación cuerpo-objeto al cuerpo de la violencia que sustenta e inaugura el siglo XXI.

\section{1. ¿Cómo comprender el paso de la violencia del cuerpo al cuerpo de la violencia?}

La perspectiva del filósofo francés, Michel Foucault sobre el poder y el cuerpo, resulta esencial para la comprensión del cuerpo (humano) y régimen disciplinario. Su puente de conexión -y éste es mi agregado a esa perspectiva- es la condición necrológica. Quizás por la mirada contrapuesta a la lectura de Giorgio Agamben y su Nuda vida. ¿Qué quiere decir esto? Significa que la vida moderna apunta, en otras palabras, a la consideración del Cuerpo como ente volcado hacia la muerte. Expresión de esto son las múltiples violencias sobre el cuerpo humano que aquí se han tratado bajo la relación Objeto-cuerpo. El control y dominio 
del cuerpo humano está transformando al mismo (cuerpo) en un territorio -permítase la analogía- del cuerpo como cementerio de disputa en la vida moderna. En consecuencia, lo que estoy diciendo es la mirada sobre el cuerpo que no es solamente un objeto "inofensivo", sino que trata de un lenguaje todavía más complejo que se relaciona con la violencia de nuestro tiempo.

El paso de la violencia del cuerpo al cuerpo de la violencia es la forma de ver cómo se ha fusionado el conocimiento humano al servicio de la muerte. Comprendemos que el conocimiento humano, técnico-científico, ha mejorado las técnicas de muerte. La muerte que se efectúa con el mínimo de costos y el máximo de rendimiento. La muerte en masa producida por la bomba atómica es un ejemplo aunque no sea el único ni el mejor. Otra técnica de muerte que se fusiona tiene que ver con el uso de armas biológicas y químicas. El filósofo alemán Peter Sloterdijk, en su libro Temblores de aire señala que las guerras se han sofisticado y que ahora se realizan en la Atmósfera. De allí que Sloterdijk lo llame Atmoterrorismo y lo considere como el no tan nuevo método y escenario del terror en el siglo XXI pero que, como bien lo explica, se realizó en la segunda guerra mundial con el uso de la mortal cámara de gas.

La sociedad disciplinaria impuesta por los Estados modernos doblegan los cuerpos. La ruptura, justamente en el momento de crisis, allí, en medio de la guerra, sale a relucir espectacularmente el cuerpo como objeto en sus tres formas. En la guerra la relación cuerpoviolencia se manifiesta con toda su sentido: el paso hacia lo que he llamado necro-lógico. No solamente porque el uso de métodos, técnicas, instrumentos y, en general, el conocimiento técnico-científico se haya puesto al servicio de la guerra y, por tanto, de la muerte, sino porque se hace evidente el régimen disciplinario establecido sobre el cuerpo. Cuerpos uniformados, enfilados, dispuestos a la muerte con otros cuerpos que siguen la misma lógica: obedecen la disciplina de la guerra. El adoctrinamiento del cuerpo. Doblegan las mentes para doblegar el cuerpo: para lograr dejarlo a merced del cuerpo de la violencia.

\subsection{Cuerpo de la violencia: la infamia de la Téchne moderna}

En definitiva es el más espeluznante desocultamiento: el sentido necro-lógico del cuerpo. El escenario de muerte. El cuerpo como un gran cementerio. En esa perspectiva, el cuerpo de la violencia se convierte en ese gran imaginario, en ese ente, un territorio por explorar, por hallar sus relieves, sus principales rupturas. El cuerpo de la violencia es la construcción de una hermenéutica de la violencia que aquí he tratado con algunos apremios. Una hermenéutica del sentido necro-lógico del cuerpo, del cuerpo de la violencia. De su forma. De la comprensión de la Téchne moderna al servicio de la muerte, de la destrucción (y también) autodestrucción de la humanidad.

Si el presente estudio tiene algún sentido, lo cobra y lo hace evidente en éste punto: en el sentido del cuerpo de la violencia. En esa infamia del conocimiento humano al servicio necro-lógico. Luego, lo que queda es un campo por explorar. Un campo por analizar acerca de esa relación Cuerpo-violencia que se ha expresado en el paso de la violencia del cuerpo al cuerpo de la violencia. En resumidas cuentas: se abre la posibilidad de comprender que la violencia tiene su propio cuerpo y que se ha expresado, precisamente, a través de los cambios sobre el cuerpo que he mencionado brevemente pero con la necesidad de decir No a la infamia de la violencia, sí al cuerpo como Sujeto-todo-vivo. 


\section{Bibliografía}

Agamben, Giorgio (2013): Homo Sacer. El poder soberano y la nuda vida, Valencia, España, Pretextos.

Foucault, Michel (1990): Tecnologías del Yo: y otros textos afines, Barcelona, España, Paidos.

Foucault, Michel (1992): Microfísica del poder, Madrid, ediciones Piqueta.

Foucault, Michel (1994): Hermenéutica del Sujeto, Madrid, ediciones Piqueta.

Foucault, Michel (2001): Defender la sociedad, México DF, Fondo de Cultura Económica. Platón (1992): República, Libro VII [Traducción de C. Eggers Lan], Madrid, Ed. Gredos. San Agustín (1985): Ciudad de Dios, Barcelona, Editorial Orbis.

Sloterdijk (2003): Temblores de aire. En las fuentes del terror [Traducción Germán Cano], Valencia, España, Pre-textos. 
